\title{
Editorial
}

\section{Nanomaterial Synthesis, Characterization, and Application}

\author{
Mahmood Ghoranneviss, ${ }^{1}$ Ajay Soni, ${ }^{2}$ Alireza Talebitaher, ${ }^{3}$ and Necdet Aslan ${ }^{4}$ \\ ${ }^{1}$ Plasma Physics Research Center, Islamic Azad University, Science \& Research Branch, Tehran, Iran \\ ${ }^{2}$ School of Basic Sciences, Indian Institute of Technology Mandi, Himachal Pradesh, Mandi 175001, India \\ ${ }^{3}$ Energy Research Institute, Nanyang Technological University, 1 CleanTech Loop, Singapore 637141 \\ ${ }^{4}$ Physics Department, Yeditepe University, 34755 Istanbul, Turkey \\ Correspondence should be addressed to Mahmood Ghoranneviss; ghoranneviss@gmail.com
}

Received 27 November 2014; Accepted 27 November 2014

Copyright (C) 2015 Mahmood Ghoranneviss et al. This is an open access article distributed under the Creative Commons Attribution License, which permits unrestricted use, distribution, and reproduction in any medium, provided the original work is properly cited.

Nanotechnology is a promising science with wide applications from cosmetics, food products, clothing, and household appliances to fuel catalyst, disease treatment, and renewable energies. Nanotechnology is also being applied to a variety of industrial and purification processes including construction materials, nanomachining of nanowires, nanorods, graphene, water filtration, and wastewater treatment.

Their applications are becoming wider in "nanomedicine" by interfacing the nanomaterials with biological molecules or structures, "green technology" to enhance the environmental sustainability and "renewable energy" to develop the new ways to capture, store, and transfer energy. For instance, carbon nanotube productions have been used for applications in energy storage, automotive parts, thin-film electronics, coatings, and so forth.

Nanomaterials are found as important and keep growing in the field of Nanoscience and Nanotechnology and in recent years researchers are investing much effort on the synthesis and applications of various nanomaterials, due to their potential applications in science and industry. For example, biocompatible nanomaterials are applied directly or they are used to replace natural materials to function or to be in contact with the living systems.

This special issue provides some new research and developments in nanomaterials. It presents the principles of the synthesis and fabrication of self-assembly nanostructures and their applications and also some important tools and challenges associated with these techniques for engineers and scientists.
First group of papers are explaining some novel approaches to enhance the optical and physical parameters of material with different techniques. For instance, one paper describes a synergistic effect combining dual-mode (down- and upconversion) with enhancement rate of the red emission in hexagonal prisms $\beta-\mathrm{NaLuF} 4: \mathrm{Er}^{3+}$ phosphors by employing the dual wavelength source. Another one describes the optimization of the parameters voltage, time, solution composition, and temperature on the properties of electrodeposited semiconductor thin films such as ECD $\operatorname{In}_{2} \mathrm{~S}_{3}$ with respect to the layer stoichiometry which can be applied to manifold optimizations.

Second group of papers are about the thin films and their physical properties. One tried to describe how monodisperse magnetic $\mathrm{Fe}_{3} \mathrm{O}_{4}$ nanoparticles can be used as the catalysts for the synthesis of vertically aligned carbon nanotube arrays and another investigated the capping layer effect of siliconnitrogen on the microstructure and electrical and optical properties of polycrystalline silicon prepared by aluminum induced crystallization and showed the improvement of the grain growth with reduction of the metal content in the induced polycrystalline silicon film.

The third group of papers addresses the new techniques of nanomaterials fabrication for electronics and magnetic application where one of them explains the synthesis of axially $\mathrm{p}-\mathrm{n}$ junction gallium nitride nanowires via metal-organic chemical vapor deposition method and shows a substantial increase in conductance under UV light exposure with extremely short response time, and the other one addresses 
the preparation of highly monodispersive Pt nanoparticles via two facile and environmental-friendly procedures which show very high catalytic activity for a methanol oxidation reaction and have a great potential for preparing other metal and alloy NPs.

Mahmood Ghoranneviss

Ajay Soni

Alireza Talebitaher

Necdet Aslan 

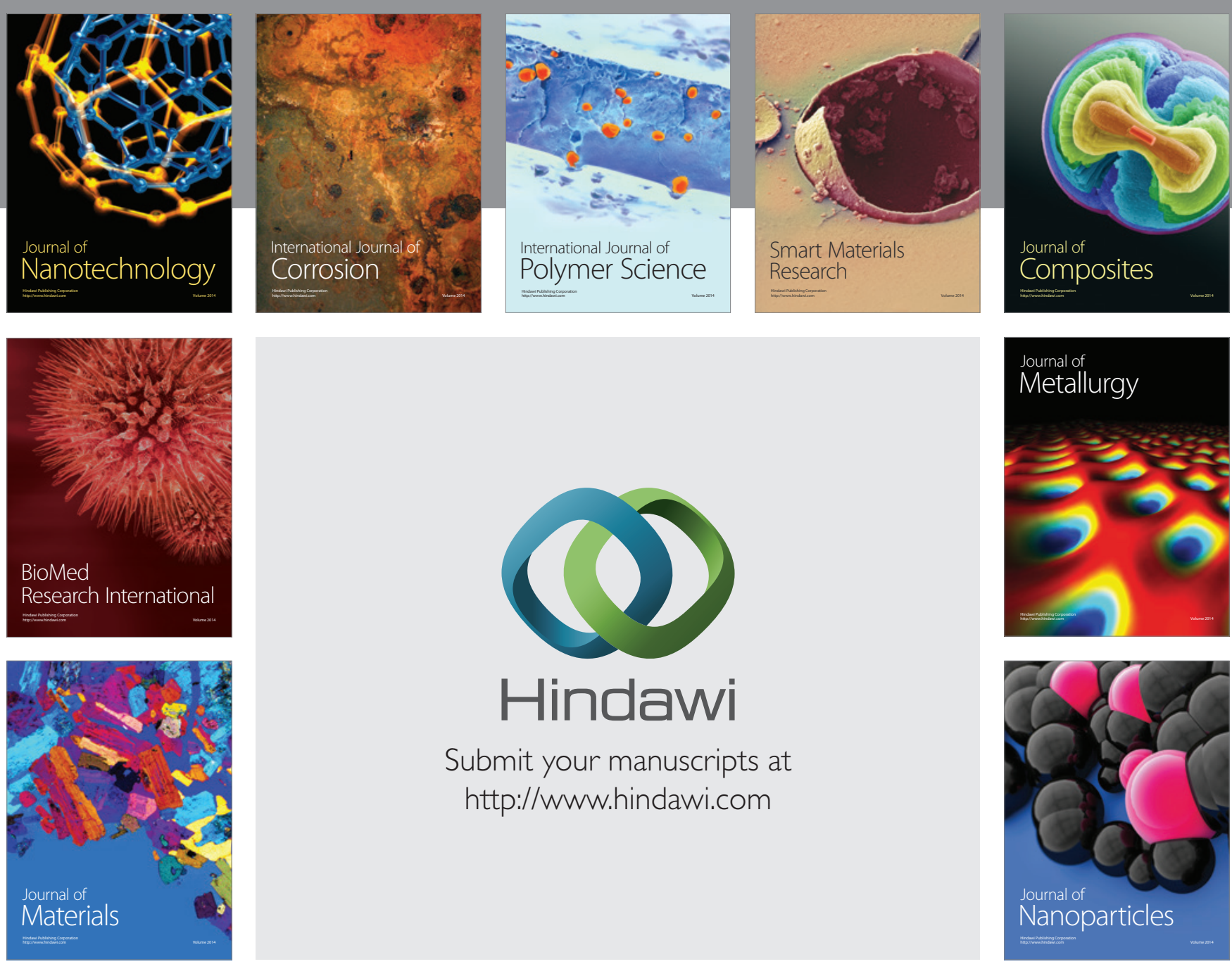

Submit your manuscripts at http://www.hindawi.com
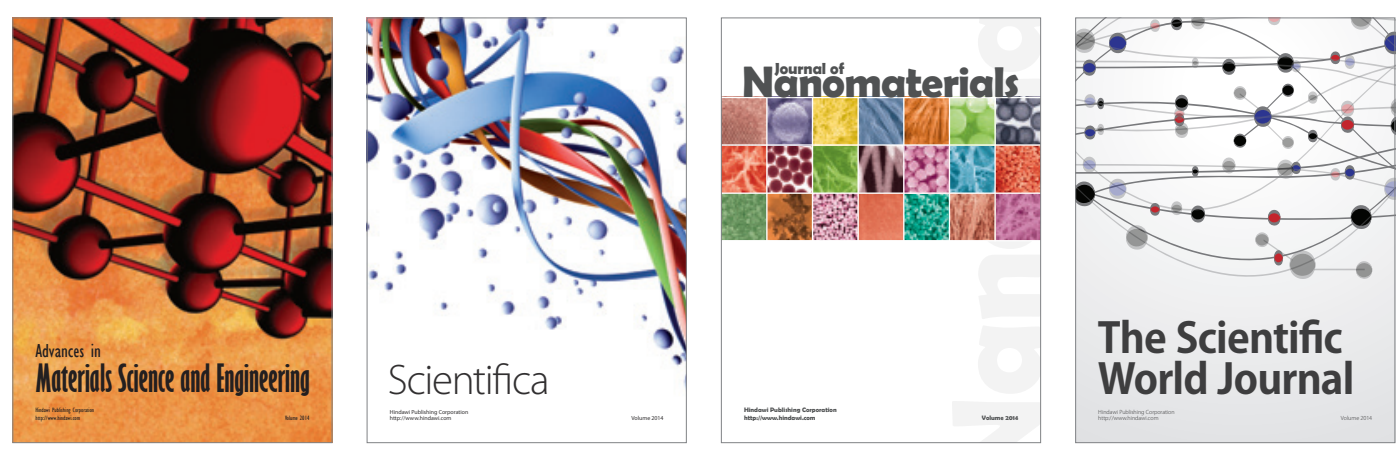

\section{The Scientific World Journal}
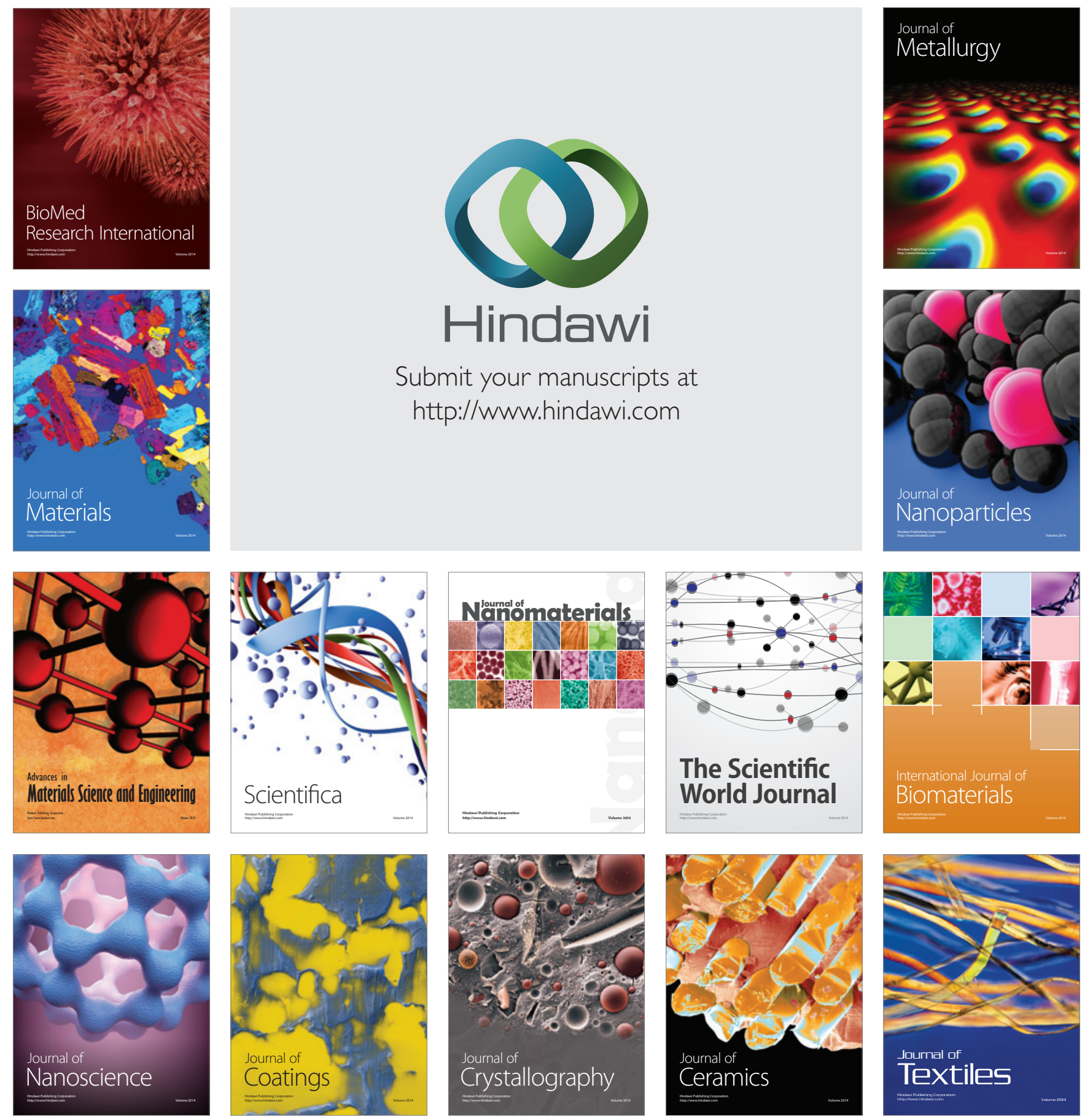